\title{
LA INDIVIDUACIÓN DESDE EL ENFOQUE DE CARL G. JUNG ${ }^{1}$
}

\section{The Individuation from the Approach of Carl G. Jung}

\author{
Juan Carlos Alonso González ${ }^{2}$
}

Universidad Nacional de Colombia

DOI: 10.17533/udea.rp.v10n1a13

Recibido: 2018-01-08 Aceptado: 2018-02-27

Para citar este artículo en APA: Alonso, J. (2018). La individuación desde el enfoque de Carl G. Jung. Revista de Psicología Universidad de Antioquia, 10 (1), 325-343.

DOI: 10.17533/udea.rp.v10n1a13

La opus humana [...] consiste en hacernos conscientes de lo que recibimos tanto a través de la biología como de la historia personal o colectiva, como del infinitamente creativo inconsciente, y en desarrollarlo de la manera óptima que nos permitan nuestras capacidades.

Murray Stein (2007, pp. 11-12).

Resumen: La Psicología Analítica de C. G. Jung considera vital para la salud mental una estrecha relación entre los niveles conscientes e inconscientes. Dicha interacción también es descrita como la relación entre el complejo personal del ego y el arquetipo colectivo del Self. Este artículo hace una revisión crítica de la literatura sobre los planteamientos de Jung y los posjunguianos, que conciben la individuación como un proceso moldeado por el Self, arquetipo de la totalidad, en el supuesto de que, cuando lo consciente y lo inconsciente, el ego y el Self, tienen una relación continua, será posible mantener un sentido de única individualidad del ser humano, así como la propia conexión con la mayor experiencia de la existencia.

Palabras claves: psicología analitica, inconsciente colectivo, ego-self, persona-sombra, personalidad.

\begin{abstract}
C. G. Jung's Analytical Psychology considered vital for mental health a close relationship between conscious and unconscious levels. Such interaction is also described as the relation between the personal complex of the ego and the collective archetype of the Self. This article makes a critical review of the literature on Jung's and postjunguian's approaches, which conceive individuation as a process molded by the Self, archetype of the totality, under the assumption that when the conscious and the unconscious, ego and Self, have a continuous relationship, it will be possible to maintain together a sense of the unique individuality of the human being, as well as he/she's own connection with the greater experience of existence.
\end{abstract}

Keywords: analytical psychology, collective unconscious, ego-self, person-shadow, personality.

1 Artículo de reflexión.

2 Psicólogo Universidad Nacional de Colombia y magíster en Estudios políticos de la Pontificia Universidad Javeriana. Analista junguiano, miembro de la International Association for Analytical Psychology (IAAP), titulado en Copenhague (2013). Miembro de la Sociedad Colombiana de Analistas Junguianos (SCAJ). Miembro fundador y Director de la Asociación de Psicología Analítica en Colombia (ADEPAC). Correo electrónico: adejungcol@yahoo.com; https://orcid.org/0000-0002-1905-2295. 
De la misma manera que no podemos escoger a nuestros padres, tampoco podemos escoger nuestra identidad ni nuestro tipo de personalidad. Padres e identidad nos son dados cuando nacemos, e influyen en la conformación de ese complejo fenómeno psicológico que llamamos el ego, compleja trama de múltiples factores conscientes e inconscientes. Stevens (1994) decía que "sólo somos libres en la medida en que adquirimos la destreza necesaria para jugar las cartas que se nos hayan repartido" (p. 67). En otras palabras, que lograremos ser más libres en la medida en que descubramos conscientemente quiénes somos y qué somos, para lo cual debemos ser capaces de escuchar y responder a una fuerza dinámica interna que nos impulsa a convertirnos en nosotros mismos. Esta búsqueda de una mayor consciencia de nosotros mismos constituye un tema de interés para la psicología junguiana, la cual ha acumulado un amplio conjunto de planteamientos e hipótesis teóricos entrelazados que aportan mucha claridad sobre este proceso de exploración interna que se ha llamado el proceso de individuación.

Un principio fundamental de la psicología de Jung es que la psique es un sistema autorregulado que busca constantemente mantener el equilibrio entre fuerzas opuestas. Tal principio plantea también que la psique tiende a comprender la realidad humana como una lucha entre fuerzas antagónicas en tensión. Se trata realmente de una "manera de pensar" (Progoff, 1967, p. 84) los fenómenos del mundo en función de oposiciones que deben ser conciliadas para lograr un desarrollo pleno. El proceso de tal unión de opuestos es la capacidad de formar por sí mismo una personalidad individual unificada, coherente, profunda y de gran riqueza.

\section{Modelo junguiano de la psique}

Como contexto general del proceso de individuación, recordemos el modelo junguiano de la psique. Aunque para Jung la psique era una especie de espacio no físico, podría ser representado, para efectos didácticos, en forma gráfica (véase Figura 1), como un gran círculo en el que una pequeña porción superior sería la conciencia; una segunda porción mayor sería el inconsciente 
personal cuyas unidades funcionales son los complejos (como el de la persona, la sombra, el anima, el animus, el complejo materno, el complejo del dinero, el complejo de poder, etc.); y en la parte inferior del círculo estaría una enorme porción que constituye el inconsciente colectivo, una de las ideas más novedosas de Jung. Jung plantea que, además de los contenidos inconscientes personales, existen otros: los arquetipos, que pertenecen a un inconsciente más amplio, y que nunca estuvieron antes en la consciencia, sino que son elementos que existen como herencia de la humanidad. El ego estaría gravitando en el umbral entre la consciencia y el inconsciente personal. Todos estos contenidos son sistemas dinámicos que participan en un proceso continuo de interacción y transformación.

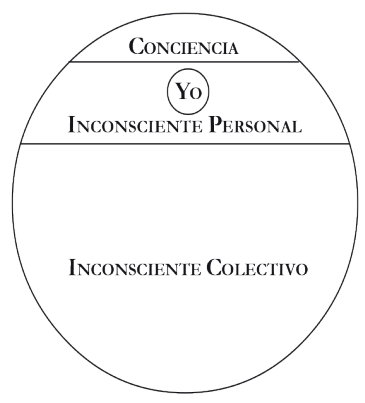

Figura 1. Modelo junguiano de la psique.

Fuente: Alonso (2004).

\section{Antecedentes del concepto de individuación}

La individuación tiene un paralelismo con el principium individuationis, el cual posee una extensa y compleja historia en la filosofía, que se remonta a Gerard Dorn (alquimista del siglo XVI), y que llega posteriormente hasta Leibniz, Locke y Schopenhauer.

La vieja escolástica había desarrollado este principio para resolver el problema relacionado con los universales. Por ejemplo, dos hombres son semejantes en tanto animales racionales, con una misma esencia; sin embargo, no se confunden en cuanto existentes, porque poseen unas características diferen- 
tes. Jung adoptó este principio de individuación, especialmente a través de Schopenhauer, para utilizarlo en el ámbito psicológico.

La forma en que lo hace la encontramos por primera vez en la definición de individuación en su obra Tipos Psicológicos (Jung, 2013, \$\$743-748)32, escrita en 1913, aunque publicada sólo hasta 1921. Menciona allí que el inconsciente colectivo representa, en la psique de la persona, lo arquetípico y específicamente humano, pero sólo de una manera potencial e indiferenciada, por lo que se requiere un proceso consciente en el cual la consciencia asimile e integre dichos contenidos colectivos, a fin de apropiárselos y hacerlos suyos, según su modo de ser único e inédito.

\section{Definición de individuación}

Vemos que existe una gran paradoja inherente a la individuación, ya que la persona se hace consciente de ser un humano particular, individual y único, y, al mismo tiempo, ser un hombre o una mujer común, semejante a otros miembros de la raza humana. Aunque las definiciones abundan, tanto a lo largo de la obra de Jung como también en la de autores posjunguianos (Samuels, 1985), nos centraremos en la definición que ofrece Jung en Tipos Psicológicos (2013). Dice que la individuación es:

el proceso por el que se constituye y singulariza el individuo, y en particular el proceso por el que se desarrolla el individuo psicológico como una entidad diferente de lo general, de la psicología colectiva. La individuación es, por ello, un proceso de diferenciación, cuya meta es el desarrollo de la personalidad individual (\$744).

El aspecto unificador de la individuación es enfatizado por su raíz etimológica. Jung (2002) precisó: “Empleo el término ‘individuación’ en el sentido de un proceso que genera un 'individuo' psicológico, es decir, una unidad, una totalidad independiente, indivisible” (\$490).

3 Es ya de uso común, con la intención de estandarizar un General Index, que las referencias a las diferentes obras de Jung se hagan indicando el número de párrafo $(\$)$ y no el número de la página de cada edición. La edición espańola que aquí se cita sigue este criterio, y en este texto también lo acogemos, salvo en los casos en los que se citen ediciones no integradas a la Obra Completa. 
Como concepto psicológico, la individuación en Jung hace énfasis en las siguientes propiedades: 1) el objetivo del proceso es el desarrollo de la personalidad; 2) ofrece un modelo para comprender e interpretar las transformaciones que se producen tanto en la psique individual como en la colectiva; 3) propone un proceso para ampliar y desarrollar la consciencia humana al máximo de su potencial (Stein, 2007, p. 11); 4) no ocurre en un estado de aislamiento del individuo, ya que presupone e incluye las relaciones colectivas; 5) este proceso puede conducir a un cierto grado de oposición a las normas sociales convencionales, las cuales no tienen una validez absoluta.

En lo que sigue del documento se desarrollarán estas propiedades.

Vínculos con el Self, ego y síntesis

El concepto de individuación está estrechamente entrelazado con otros conceptos, principalmente con ego, Selfy sintesis.

Jung definió al ego como el centro de la consciencia, resaltando sus limitaciones y diferencias con la personalidad total. El ego se relaciona con cuestiones tales como la identidad del individuo, la conservación de su personalidad, la persistencia a través del tiempo, el conocimiento de la realidad y la mediación entre el reino consciente e inconsciente. No obstante, es una instancia psíquica que responde a las necesidades de otra que le es superior: el Self, principio ordenador de la personalidad entera (Samuels, Shorter \& Plaut, 1997). La relación del Self con el ego puede ser comparada con la relación entre lo que mueve y lo que es movido.

El proceso de individuación, conscientemente perseguido, lleva a la percepción del Self. Por eso, la individuación es esencialmente diferente del simple proceso de tomar consciencia (Sharp, 1994, p. 109). Astor (1995) dice que "el ego es a la integración (vista socialmente como adaptación), lo que el Selfes a la individuación (auto-experiencia y auto-realización)” (p. 231).

Jung insistía repetidamente en diferenciar el proceso de individuación con la simple toma de consciencia del ego, lo cual llevaba a identificar al ego con el Self, causando así grandes confusiones conceptuales: por ejemplo, el error de asimilar la individuación con el egocentrismo. Reiteraba, entonces, que el Self abarca una infinidad de aspectos relacionados con la trascendencia de la 
vida, mucho más profundos que las que preocupaciones cotidianas que suele contener el ego.

Respecto al concepto de sintesis, la individuación tiene como objetivo lograr la síntesis óptima de los procesos y fantasías conscientes e inconscientes. Esto conduce a la persona no sólo a experimentar su propia particularidad individual, sino también a reconocer que existen fuerzas dentro y fuera de ella, que trascienden su comprensión personal y consciente. De ahí que, aunque el proceso de individuación incluye el proceso de individualización, lleva a la persona más allá de ese proceso esencial de creación del ego, hacia la búsqueda de valores, sentido y autotrascendencia (Gordon, 2000). Jung (2002) afirma que "la meta del proceso de individuación es la síntesis del Self" (\$278).

\section{Fases del proceso de individuación}

Aunque las fases más significativas del proceso de individuación no fueron determinadas con precisión por Jung, hay otros autores (Jacobi, 1963; Von Franz, 1982) que han tratado de recoger de manera más ordenada algunos de los principales temas junguianos, dispersos a lo largo de su obra completa. Con el riesgo de pecar de esquemático, recojo las etapas propuestas por Vázquez (1981, p. 267) acerca de este proceso interior de autorrealización de la personalidad. Este toma como modelo referencial el mito del héroe, tan importante en Jung, con el que se puede describir el difícil y peligroso camino que debe emprender el héroe o la heroína en búsqueda de la conquista del Self, cual tesoro escondido, a sabiendas de los enormes escollos y ordalías con las que se deberá enfrentar. Las fases son las siguientes: 1) llamada o vocación; 2) consciencia de la llamada; 3) decisión moral o respuesta a la llamada; 4) desalienación parental; 5) desalienación social o retiro de la máscara; 6) integración de la sombra; e 7) integración del anima-animus.

Aunque no podemos describir en detalle estas fases, quisiera hacer unos comentarios generales sobre algunos aspectos centrales de esta trayectoria: la llamada, la desalienación, la máscara, la sombra y la integración. La "llamada", que puede ser generada por diferentes causas, llevaría al individuo a un 
conflicto o desarmonía interna entre pares de opuestos de la personalidad, que se puede manifestar externamente en la sensación de que hace falta algo en la vida. Esta llamada no parecería provenir del ego, sino de la voz interior del Self.

La desalienación se refiere mitológicamente no sólo al alejamiento del héroe de sus padres, sino también a la salida de su ciudad. Es decir, para avanzar en la individuación, se requiere que el individuo supere la alienación, tanto de las fijaciones parentales como de las sociales, diferenciándose de la persona o máscara social, con la cual se ha debido identificar el yo en su adaptación al mundo exterior.

El tema de la sombra es importante para diferenciar la individuación junguiana de otros procesos de autorrealización, propuestos por enfoques diferentes, porque el objetivo de esta no es dominar la propia psicología personal ni llegar a ser perfecto, tampoco a ser "más bueno", sino familiarizarse con su psicología y tratar de ser "más completo" e integrado. De ahí que la individuación tenga esa fase interna y subjetiva de integración de la sombra, del anima y del animus, lo que implica una creciente percepción de nuestra realidad psicológica única, incluyendo fortalezas y limitaciones personales, y al mismo tiempo una apreciación más profunda de la humanidad en general (Sharp, 1994, p. 107). En otras palabras, en la medida en que nos veamos más humanos, podremos ver humanizados a quienes nos rodean.

Las anteriores fases de múltiples integraciones entre opuestos (ego-Self; persona-sombra; anima-animus, entre otros) son posibles durante la individuación, a través del operar del Self, que permite que el individuo logre liberarse gradualmente de tales fuerzas contrarias mediante una solución simbólica ("una suspensión de la voluntad"), llamada la función trascendente del símbolo, que les permite tener la completa igualdad (Astor, 1995, p. 231). Al respecto, afirmaba Jung (2013):

Cuando existe la plena paridad de los opuestos, atestiguada por la participación absoluta del ego en ambos, esto necesariamente conduce a una suspensión de la voluntad, porque la voluntad ya no puede operar, cuando todo motivo tiene un contra-motivo igualmente fuerte. Puesto que la vida no puede tolerar un punto muerto, resulta un represamiento de energía vital, y esto llevaría a una condición 
insoportable si la tensión de los opuestos no produjera una nueva función unificadora que los trasciende (\$824).

En ese proceso dialéctico en el que participa tanto lo inconsciente como la consciencia, la individuación se manifiesta a través de dos formas fundamentales:

Un proceso de individuación natural inconsciente, el cual ocurre en el ser humano como un simple acontecimiento metafísico, con el mismo sentido que tiene para una bellota llegar a ser un árbol de roble. La consciencia no interviene en absoluto; los símbolos arquetípicos individualizadores se dan naturalmente, sin que el individuo sea consciente de su proceso de realización y transformación de su propia personalidad.

Un proceso de individuación consciente, netamente humano, en el que la consciencia tiene que confrontarse con lo inconsciente y encontrar un equilibrio entre los contrarios, mediante símbolos producidos espontáneamente por el inconsciente y amplificados por la consciencia, provenientes del Self. De esta forma, el diálogo entre el inconsciente y la consciencia hace que, de una parte, la personalidad quede iluminada, y de otra, que la consciencia se amplíe y gane en amplitud de visión. Este es el "fenómeno de realización individual" que hace que todo ser humano que avance en su proceso de individuación, conozca mejor su propio ser más íntimo y auténtico, sobre el cual tenía antes una verdadera ceguera psicológica (Vázquez, 1981, pp. 255-256).

Esto último significa que, en este proceso de desarrollo interior, los contenidos arquetípicos provenientes del inconsciente colectivo tienden a actualizarse a través de la consciencia particular,

buscando una conformación individual en la vida y en la obra de cada uno (...). Por su oposición a la consciencia, no pueden sin embargo, ser trasladadas inmediatamente a nuestro mundo. Así, hay que buscar un camino que abra comunicación entre la realidad consciente y la inconsciente (Jung, 1992, p. 95).

Eso equivale a decir que no hay dos caminos de individuación iguales, sino que cada persona debe buscar su propia senda absolutamente particular y única para llegar a ser él mismo. 


\section{Adaptación interna y externa}

Dichas sendas particulares nunca podrían alcanzarse por el camino prescrito por las normas colectivas, por lo que el individuo debe lograr adaptarse tanto a su vida externa como a su mundo interno. Es decir, para que le sea posible la individuación, se requiere, por una parte, que el individuo haya logrado, primero, un mínimo de adaptación a las normas colectivas; pero, por otra parte, dicha adaptación no se consigue sin que exista una cierta contraposición a ellas; por cuanto la individuación supone una orientación distinta, como diferenciación de lo general y formación de lo peculiar, ya que coincide con el desarrollo de la consciencia desde su original estado de identidad (Vázquez, 1981, p. 253).

Todo esto se aclara al tener en cuenta el concepto de individuo, cuya definición nos la ofrece Jung, también en Tipos Psicológicos (2013): "El individuo psicológico se caracteriza por poseer una psicología singular y en cierto sentido única” (\$749). La individualidad inconsciente tiene un carácter colectivo generalizador e indiferenciado, por lo que se necesita un proceso consciente de diferenciación, para hacer consciente tal individualidad, es decir, para resaltar la diferencia con respecto a los demás individuos.

Considero importante resaltar este tema de la individuación y la adaptación al mundo externo, pues existe una falsa creencia, según la cual el proceso pone énfasis en la adaptación del ego a su mundo interno y niega o descuida el mundo exterior. Por el contrario, Jung (2013) afirmaba:

como el individuo no es sólo una entidad singular, sino que su misma existencia presupone la relación con una colectividad, el proceso de individuación no conduce al aislamiento, sino a una cohesión colectiva aún más intensa y universal (\$744).

Y añade: "La individuación no nos cierra las puertas del mundo, sino que reúne el mundo para sî" (Jung citado por Sharp, 1994, p. 107).

La anterior reflexión lleva también a otra, relacionada con la diferencia entre individualismo e individuación, pues tiene nexos de similitud, aunque también otros que marcan una gran diferencia. 


\title{
El individualismo frente a la individuación
}

En el enfoque junguiano, el proceso de individuación no se refiere solamente a la descripción del proceso por el cual la personalidad se va formando en el transcurso de las distintas etapas de la vida (infancia, juventud y adultez), pues esto equivaldría a la suma de las variables biogenéticas, psicogenéticas y culturales; y si tal fuese, la individuación sería muy semejante al individualismo. El individualismo significa destacar deliberadamente y dar prominencia a alguna supuesta particularidad del individuo, más que a las consideraciones y obligaciones colectivas. Por el contrario, la individuación promueve que la consciencia vaya más allá de los límites a los que llegan los procesos normales del desarrollo regidos por el individualismo.

Jung (2013) afirmaba:

\begin{abstract}
Con la norma colectiva sólo se suscita un conflicto real cuando se eleva una vía individual a la categoría de norma, cosa que constituye el auténtico propósito del individualismo radical. Como es natural, este propósito es en sí mismo patológico y contrario a la vida, por lo que no tiene en realidad nada que ver con la individuación, la cual precisamente por tomar un camino lateral individual, precisa también de la norma para orientarse frente a la sociedad y establecer con ella esa relación de la que el individuo tiene vital necesidad. La individuación conduce, por ello, a que las normas colectivas sean objeto de una estima natural (\$747).
\end{abstract}

En otras palabras, la individuación se desvía de las normas colectivas, aunque las siga respetando; mientras que el individualismo las evita totalmente (Sharp, 1994, p. 109). En la individuación, la desviación de lo colectivo se da cuando la persona busca que la consciencia se aleje de sus hábitos personales establecidos y de sus actitudes condicionadas por la cultura, para conducirlas hacia un horizonte impersonal mucho más amplio de comprensión de uno mismo, con lo cual se produce una ampliación de la consciencia, que la lleva más allá de lo personal, hacia lo arquetípico (Stein, 2007, p. 13). 


\section{La ética individual frente a la ética colectiva}

Otro aspecto que se desprende de la individuación es la ética. Para la psicología junguiana, el desarrollo pleno de la personalidad que se presenta en la individuación supone "una decisión moral cuya perentoriedad sólo llega a percibir quien se ve ante la necesidad de decidirse sobre la asimilación de lo inconsciente y la integración de su personalidad” (Jung, 1970, p. 153). Jung va a insistir una y otra vez en la necesidad de incluir los valores éticos en la psicología, si se quiere llegar a la personalidad total del individuo. No se trata, sin embargo, de la ética tradicional y externa, sino de la fidelidad a sí mismo, que se manifiesta como una llamada o una voz interior.

Jung era enemigo de la pertenencia a los grupos grandes, porque tenía el convencimiento de que éstos producían una disminución de la responsabilidad y de la ética individual, ya que el individuo tendía a ver que sus obligaciones y deberes eran absorbidos por la ética colectiva de los grupos grandes. Decía: "Cuanto más fuertes son las normas colectivas que gobiernan la vida de las personas, tanto mayor es su inmoralidad a nivel individual” (Jung, 2013, $\$ 747)$.

Neumann (1960) habla de una "ética de individuación", y la relaciona con la labor de integración de la sombra. Complementa lo dicho por Jung, afirmando que el "trabajo con la sombra", que lleva al individuo a la comprensión de su propia imperfección, lo conduce igualmente a liberarse de una identificación con los valores colectivos. Añade que la personalidad que ha adquirido tal autonomía ética,

es un polo de reposo en el fluir de las olas del colectivismo y de la psique de las masas. Estas olas sólo logran arrastrar consigo a la personalidad que está parcialmente desarrollada desde el punto de vista ético por no anclar sus raíces en lo inconsciente (Neumann, 1960, p. 119).

En el mismo sentido, Gordon (2000) afirma que la individuación implica una disposición para la cada vez mayor conciencia de la propia naturaleza de cada uno, con sus tendencias y cualidades tanto positivas como negativas, lo que lleva a una comprensión cada vez más clara de lo que uno es. Por lo tanto la 
auto-imagen se hace cada vez más adecuada y se acompaña de un creciente reconocimiento de la propia separación y responsabilidad personal de cada uno (pp. 175-176).

\section{Interrogantes sobre la individuación}

En una revisión crítica de los planteamientos junguianos sobre la individuación, Samuels, Shorter y Plaut (1997) se plantean un interrogante, que consideran sin respuesta aún, dirigido a saber si la integración de los opuestos debe ser un requisito previo para la individuación. Es obvio que, si la conciliación de opuestos se lleva a cabo con antelación, las oportunidades futuras que tiene el ego fuerte (integrado) para resistir las tensiones del camino de la individuación serán mejores, una vez que emerge la etapa del "llamado" del inconsciente. Mencionan que, aunque existen renombrados artistas, como van Gogh, Mozart o Gauguin, que indudablemente lograron una autorrealización, difícilmente se podría decir de ellos que lograron una integración de su personalidad, pues a veces parecerían haber quedado fijados con características infantiles o con rasgos psicóticos. ¿Lograron ellos un proceso avanzado de individuación? Samuels et al. (1997) mencionan que la respuesta sería afirmativa en términos de perfección de sus ingenios, que se fusionaron con sus personalidades; pero probablemente no en términos de completitud, adaptación social y relaciones interpersonales.

Otra pregunta relativa a la individuación, que no se restringe a la psicología junguiana, sino que concierne a toda psicología profunda, es la de si habrá alguna diferencia para el resto de la humanidad, si un número infinitamente pequeño emprende este arduo trabajo. Jung opinaba que ello tendría un gran impacto, ya que el analista no estaba trabajando solamente para el paciente, sino también para su propio proceso interno, y añadía que "aunque su aportación sea pequeña e invisible, es un opus magnum... Las preguntas últimas y supremas de la psicoterapia no son un asunto privado, sino una responsabilidad ante una instancia suprema” (Jung, 2006, \$449).

Otro aspecto que se ha debatido bastante es si la individuación está reservada sólo para unas pocas personas, de características especiales. El mismo 
Jung puede haber contribuido a esta idea, afirmando explícitamente que el proceso de individuación es una ocurrencia relativamente rara. Además, los ejemplos de individuación que se presentan en su obra parecen corresponder a personalidades excepcionales. Todo ello, llevó a la noción de que se estaba refiriendo a un concepto elitista.

De las tres escuelas que se suelen distinguir en el enfoque junguiano (Samuels, 1999), la arquetipal, la evolutiva y la clásica, es quizás esta última la que puede conservar la idea de que la individuación no es para todo el mundo. Por el contrario, Samuels explora algunas coincidencias entre las otras dos escuelas (arquetipal y evolutiva) y encuentra una especie de revisionismo, al coincidir las dos en la necesidad de "operacionalizar" y bajar a tierra la idea de la individuación. De una parte, se analiza que es un proceso que puede tener su inicio en cualquier momento de la vida, aun desde la niñez. De otra parte, que no es desarrollable sólo por personas especialmente evolucionadas, sino que los psicológicamente "normales" y aun los débiles son capaces de beneficiarse de este proceso (Alonso, 2004; Sáinz, 1991). Concretamente, dentro de la escuela evolutiva, el concepto de individuación se convirtió en un concepto clave de la contribución de Jung a las teorías del desarrollo de la personalidad. La afirmación de Jung sobre que la individuación formaba parte de la segunda mitad de la vida, fomentó el más importante desarrollo teórico sobre este concepto, el cual se hizo extensivo para pasar a comprender también el comienzo de la vida (Fordham citado por Samuels et al. 1997, p. 75).

Algunos de los autores posjunguianos han considerado que, aunque Jung eligió ejemplos extremos para demostrar la individuación con mayor facilidad, lo cierto es que creía que el proceso podía ocurrir en circunstancias más moderadas (Samuels et al. 1997). La experiencia muestra que la transformación puede comenzar a partir de un evento con una gran carga emocional (como el nacimiento, la muerte, una separación); sin embargo, también puede generarse en el consultorio de un terapeuta, como resultado de un proceso técnico. En ese último caso, un trabajo adecuado de los fenómenos transferenciales puede ser de fundamental importancia, como se verá luego.

Individuación durante el análisis 
En opinión de Jung, uno de los propósitos del análisis junguiano, quizás el principal, es ayudar al proceso de individuación, principalmente en el plano arquetípico. Aunque, como se mencionó anteriormente, varios consideran que la individuación puede comenzar a cualquier edad, es claro que la emergencia de su necesidad suele ocurrir con más frecuencia durante la segunda mitad de la vida. Esto tiene sentido, si se tiene en cuenta que, durante la primera mitad de la vida, el individuo tiende a estar más centrado en crear, en cierta medida, una adecuada persona o "máscara social" que le permita adaptarse a su mundo externo, así como a fortalecer su ego, lo cual será de máxima importancia para resistir las presiones que demanda la emergencia de contenidos inconscientes durante la individuación. Dice Stein (2004):

La individuación incluye algo más que el proyecto idealmente logrado en la primera mitad de la vida, a saber, el desarrollo del ego y de la persona... Una vez que esto ha sido logrado, comienza a manifestarse otra tarea porque el desarrollo ideal del ego y de la persona ha dejado fuera del marco de la consciencia una gran cantidad de material psicológico. La sombra no ha sido integrada, el anima y animus permanecen inconscientes y aun cuando ha estado actuando desde el trasfondo, el Self apenas ha podido ser vislumbrado directamente (p. 228).

\title{
Y ańade luego:
}

\begin{abstract}
Ahora la tarea consiste en unificar el yo con el inconsciente que contiene la vida no vivida de individuo y su potencial no realizado. Este desarrollo en la segunda mitad de la vida sería el significado junguiano clásico de la individuación de convertirse en lo que uno potencialmente ya es, pero ahora más profunda y más conscientemente. Esto requiere de la fuerza facilitadora de los símbolos que revelan contenidos inconscientes que descansaban en la oscuridad haciéndolos asequibles para la consciencia (Stein, 2004, p. 231).
\end{abstract}

Muchos temas tratados durante un proceso de análisis pueden no estar estrictamente centrados en facilitar la individualización. Aunque en el pasado se hacía una distinción entre terapia de apoyo, en la cual se trabajaban circunstancias externas difíciles del paciente, mientras que la terapia analítica se centraba propiamente en la individuación, la verdad es que, en la práctica clínica actual, se trabajan los dos aspectos durante un proceso analítico junguiano, 
entre otros motivos, porque es indudable que la individuación puede ayudar indirectamente a solucionar los problemas cotidianos (Zoja, 1989, p. 331). Sin embargo, es claro que la prioridad del análisis es el fomento del proceso de individuación del paciente a través de la exploración y la experiencia de los símbolos y figuras arquetípicos en sueños, visiones, imaginación activa y en la vida cotidiana (Hopcke, 1992, p. 57).

Recapitulando lo dicho hasta el momento, la senda de la individuación procura, en esencia, un proceso de recentramiento de la propia personalidad, la cual tiende a ser desplazada de su verdadero centro durante la primera mitad de la vida, lo que a su vez provoca una alienación del ego, que se suele identificar con una de sus funciones, la persona o "máscara social". Progresar en la individuación, en este sentido, es encontrarse a sí mismo (Vázquez, 1981, p. 257). Por eso, los sueños con mandalas sugieren el simbolismo del Selfsiempre que aparecen un centro y un círculo. También se está atento a la aparición de bodas, que pueden simbolizar la individuación como una relación matrimonial. Estos son unos pocos ejemplos específicos de contenidos de naturaleza interpersonal que se prestan para el trabajo de individuación.

Respecto a la metodología, la transferencia en el análisis desempeña un papel decisivo para la individuación. La transferencia no puede ser inducida por el analista ni mucho menos ser exigida. La labor del analista junguiano es simplemente crear un ambiente confiable y seguro de contención, que facilite el proceso. Volviendo a la mencionada diferencia entre terapia de apoyo y terapia analítica, en esta última prima la personalidad del analista más que una determinada técnica correcta. Esto significa que más que conocimientos sobre la individuación e intuiciones acerca a partir de su experiencia personal, el terapeuta debe tener la mente abierta a las producciones inconscientes del paciente, que pueden ir desde síntomas físicos hasta sueños, visiones o pinturas, para intuir su posible significado.

Por último, se puede hablar de una psicopatología de la individuación en el sentido de los peligros normales que se pueden presentar durante un proceso de individuación. El primero que advertía Jung era la inflación del ego, producto de la asimilación de contenidos inconscientes en la consciencia, y cuya consecuencia puede ser una desorientación acompañada de un sentimiento de 
inmenso poder y singularidad, o de un sentido de desvalorización. El primero representaría un estado hipomaníaco, mientras que el segundo sería de depresión (Jung, 2007). Otro peligro podría residir en los efectos que pueden tener la emergencia de contenidos inconscientes en personas de poco contacto con la realidad, y que podrían conducir a colapsos esquizofrénicos. Jung se refiere a las ideas psicóticas que pueden ensoberbecer al ego. Asimismo, Samuels et al. (1997) previenen sobre el peligro de una implicación intensa con el mundo interior y sus imágenes fascinantes en el caso de personalidades narcisistas.

\section{Conclusión}

La psicología junguiana, a semejanza del psicoanálisis freudiano, busca dominar la represión y transferir contenidos inconscientes de la sombra personal hacia la consciencia, y restar al ego la creencia de absoluta estabilidad y de ser centro privilegiado del universo psíquico. No obstante, se considera que el enfoque junguiano va más allá que el psicoanálisis, al proponer el establecimiento de una comunicación constante con el espíritu del inconsciente colectivo. Se busca, además, un nivel más profundo que el que trata de revelar la psicología del ego. El enfoque parte de la convicción fundamental de que los seres humanos estamos evolucionando en cuanto a la ampliación de nuestra consciencia, tanto individual como colectivamente, y que podemos tener un papel activo en este proceso. Este enorme reto exige una ardua labor psicológica, cuyo fin último es la ampliación de la conciencia y las múltiples integraciones en los planos somático, psicológico y espiritual del ser humano, y tanto en lo individual como en lo colectivo (Stein, 2007).

La individuación representa un auténtico desarrollo de la personalidad, que busca que el in-dividuo llegue a hacerse él mismo, de una forma indivisible, en una totalidad integrada de todos sus componentes internos, armónica entre sus dualidades opuestas y diferente de la psicología colectiva. Esto último implica tanto despojarse a sí mismo de los falsos atuendos de la persona o máscara social como diferenciarse psicológicamente de lo colectivo, llegando a ser distinto de los demás seres humanos, pero sin aislarse, manteniendo una relación con ellos (Jung, 2007, \$269). 
Mientras la meta sea la totalidad y la sana interacción de trabajo con el Self, el verdadero valor de la individuación reside no en la meta final, sino en lo que va ocurriendo en el camino (Sharp, 1994, p. 110). Según Jung, nadie está nunca completamente individuado. Esto equivale a decir que la individuación no es sino un objetivo en potencia, cuya idealización es más fácil que su realización, que "la meta sólo es importante como idea; sin embargo, lo esencial es el opus que conduce a la meta, pues llena con un sentido la duración de la vida” (Jung, 2006, \$400). La individuación es un proceso dinámico que dura toda la existencia. 


\section{REFERENCIAS}

Alonso, J. C. (2004). La Psicología Analítica de Jung y sus aportes a la psicoterapia. Universitas Psychologica, 3 (1), 55-70.

Astor, J. (1995). Michael Fordham: Innovations in Analytical Psychology. London: Routledge.

Gordon, R. (2000). Dying and Creating a Search for Meaning. London: Karnac.

Hopcke, R. (1992). A Guided Tour of the Collected Works of C. G. Jung. Boston-London: Shambhala.

Jacobi, J. (1963). La psicología de C. G. Jung. Madrid: Espasa-Calpe.

Jung, C. G. (1970). Arquetipos e inconsciente colectivo. Buenos Aires: Paidós.

Jung, C. G. (1992). Lo Inconsciente: en la vida psíquica normal y patológica. Buenos Aires: Losada.

Jung, C. G. (2002). Los arquetipos y lo inconsciente colectivo. En Obra Completa (Vol. 9/1). Madrid: Trotta.

Jung, C. G. (2004). La dinámica de lo inconsciente. En Obra Completa (Vol. 8). Madrid: Trotta.

Jung, C. G. (2006). La práctica de la psicoterapia. Contribuciones al problema de la psicoterapia y a la psicología de la transferencia. En Obra Completa (Vol. 16). Madrid: Trotta.

Jung, C. G. (2007). Dos escritos sobre Psicología Analítica. En Obra Completa (Vol. 7). Madrid: Trotta.

Jung, C. G. (2013). Tipos Psicológicos. En Obra Completa (Vol. 6). Madrid: Trotta. 
Neumann, E. (1960). Psicología Profunda y nueva ética. Buenos Aires: Compañía General Fabril Editora.

Progoff, I. (1967). La psicología de C. G. Jung y su significación social. Buenos Aires: Paidós.

Sáinz, F. (1991). La psicología de Carl Jung en el mundo. Revista de Psicología de El Salvador, 10 (41), 1-7.

Samuels, A. (1985). Jung and the Post-Jungians. London: Routledge.

Samuels, A. (1999). Jung y los postjunguianos. En P. Young-Eisendrath \& T. Dawson (Eds.), Introducción a Jung (pp. 39-54). Madrid: Cambridge University Press.

Samuels, A., Shorter, B. \& Plaut, F. (1997). A Critical Dictionary of Jungian Analysis. London: Routledge.

Sharp, D. (1994). Lexicón Junguiano. Santiago de Chile: Cuatro Vientos Editorial.

Stein, M. (2004). El mapa del alma según Jung. Barcelona: Ediciones Luciérnaga.

Stein, M. (2007). El principio de individuación: hacia el desarrollo de la conciencia humana. Barcelona: Ediciones Luciérnaga.

Stevens, A. (1994). Jung o la búsqueda de la identidad. Madrid: Debate.

Vázquez, A. (1981). Psicología de la Personalidad en C. G. Jung. Salamanca: Sígueme.

Von Franz, M.-L. (1982). C. G. Jung: su mito en nuestro tiempo. México: Fondo de Cultura Económica.

Zoja, L. (1989). Working against Dorian Gray: analysis and the old. En S. Andrew (Ed.), Psychopathology: Contemporary Jungian Perspectives (pp. 327-345). London: Karnak Books. 
